\title{
Less invasive surfactant administration versus endotracheal surfactant instillation followed by limited peak pressure ventilation in preterm infants with respiratory distress syndrome in China: study protocol for a randomized controlled trial
}

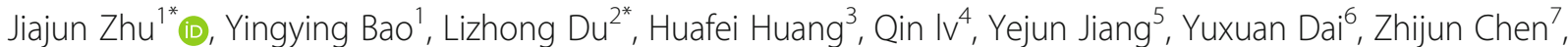
Jingyun Shi ${ }^{8}$, Yongyan Shi ${ }^{9}$, Chuangzhong Yang ${ }^{10}$, Hua Mei ${ }^{11}$, Hong Jiang $^{12}$, Yanhui Sun ${ }^{13}$ and Xuemei Sun ${ }^{14}$

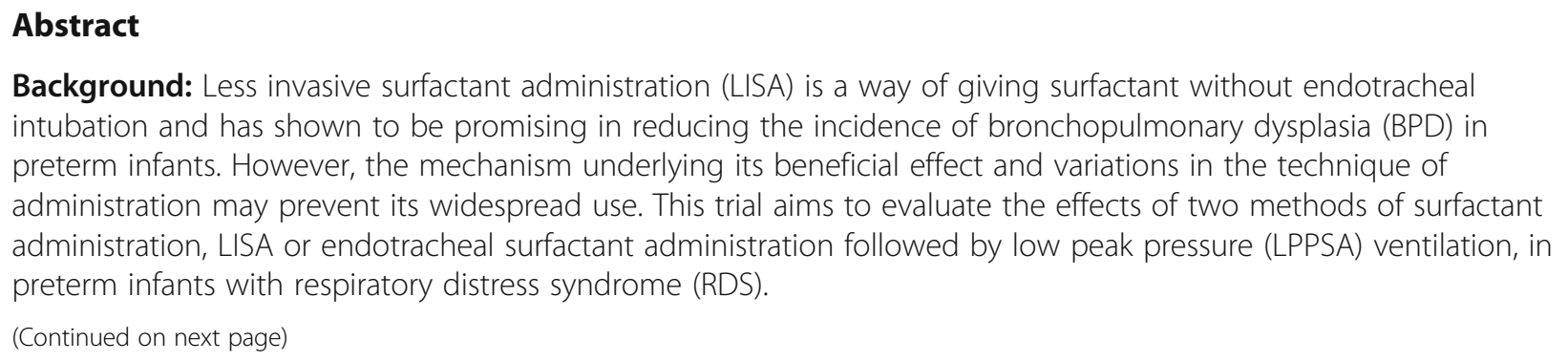

\footnotetext{
* Correspondence: jiajunzhu@zju.edu.cn; dulizhong@zju.edu.cn

The $4^{\text {th }}$ to $14^{\text {th }}$ author's list is not ranked.

'Women's Hospital, Zhejiang University, School of Medicine, Hangzhou

310006, China

${ }^{2}$ The Children's Hospital, Zhejiang University, School of Medicine, Hangzhou

310052, China

Full list of author information is available at the end of the article
}

(c) The Author(s). 2020 Open Access This article is licensed under a Creative Commons Attribution 4.0 International License, which permits use, sharing, adaptation, distribution and reproduction in any medium or format, as long as you give appropriate credit to the original author(s) and the source, provide a link to the Creative Commons licence, and indicate if changes were made. The images or other third party material in this article are included in the article's Creative Commons licence, unless indicated otherwise in a credit line to the material. If material is not included in the article's Creative Commons licence and your intended use is not permitted by statutory regulation or exceeds the permitted use, you will need to obtain permission directly from the copyright holder. To view a copy of this licence, visit http://creativecommons.org/licenses/by/4.0/ The Creative Commons Public Domain Dedication waiver (http://creativecommons.org/publicdomain/zero/1.0/) applies to the data made available in this article, unless otherwise stated in a credit line to the data. 
(Continued from previous page)

Methods: The LISA Or Low Peak Pressure trial is to be conducted in 14 tertiary neonatal intensive care units in China. A total of 600 preterm infants born with gestational age between $25^{0 / 7}$ and $31^{6 / 7}$ weeks and with a primary diagnosis of RDS will be involved in the study. Infants will be randomized to the LISA or LPPSA group when surfactant therapy is indicated. Primary outcomes include mortality, severity of bronchopulmonary dysplasia at 36 weeks of postmenstrual age (PMA), and mechanical ventilation (MV) in the first $72 \mathrm{~h}$ of life. Secondary outcomes include the days of MV, duration of all sorts of non-invasive respiratory support, fraction of inspired oxygen, oxygen saturation before and after surfactant administration, and time required to perform the procedure for surfactant administration. The incidence of comorbidities, including retinopathy of prematurity (ROP), necrotizing enterocolitis (NEC), intraventricular hemorrhage (IVH), hemodynamically significant patent ductus arteriosus (hsPDA), pneumothorax, and massive pulmonary hemorrhage within $48 \mathrm{~h}$ of surfactant administration, and the failure rates of each technique will be determined.

Discussion: Data from recent systematic review and meta-analysis have suggested a possible improvement in outcomes of preterm infants with RDS by the LISA technique. However, robust evidence is lacking. Why LISA plays a potential role in reducing respiratory morbidity, mainly BPD in preterm infants, remains unclear. The possible explanations are the active and uninterrupted delivery of continuous positive airway pressure during the LISA procedure and the avoidance of complications caused by intubation and relatively high pressure/volume ventilation following surfactant administration. We hypothesized that LISA's effectiveness lies mainly in avoiding relatively high-pressure positive ventilation immediately following surfactant administration. Thus, this multicenter randomized controlled trial will focus on issues of endotracheal intubation and the pressure/volume used during conventional surfactant administration. The effectiveness, safety and comorbidities of preterm infants following LISA or LPPSA will be evaluated.

Trial registration: Chinese Clinical Trial Registry: ChiCTR1900020970. Registered on 23 January 2019.

Keywords: Less invasive surfactant administration, Low peak pressure ventilation, Respiratory distress syndrome, Preterm infant

\section{Background}

In the last several years, dramatic improvements have been made in the management of respiratory distress syndrome (RDS) in preterm infants, including early establishment of FRC by noninvasive respiratory support, early rescue instead of prophylactic surfactant use, and curtailed application of mechanical ventilation (MV). All these changes have greatly improved the outcome for babies with RDS [1, 2].

Less invasive surfactant administration (LISA), as an innovative mode of surfactant delivery, is getting increasing attention in the management of preterm infants with RDS. This technique is characterized by the use of a thin catheter to deliver surfactant instead of an endotracheal tube and by keeping continuous positive airway pressure (CPAP) support during the course of administration [3]. A recent systemic review and meta-analysis in preterm infants with RDS indicated that surfactant administration via the LISA method decreased the combination rate of mortality or bronchopulmonary dysplasia (BPD) and reduced need for MV during the first $72 \mathrm{~h}$ of life [4]. However, the implementation of the technique requires an experienced clinician and consistence when performing the procedure. Moreover, the mechanisms underlying the beneficial effect of LISA over conventional methods are not fully understood, and further research is warranted [5]. While the intubationsurfactant-extubation (INSURE) technique as a conventional method has been widely used, evidence on superiority, efficacy, and safety of the LISA method over conventional surfactant administration are needed.

To provide more evidences and elucidate the potential protective mechanisms of the LISA method, we therefore conduct a multicenter, prospective, randomized controlled trial in China. This trial aims to compare the effects of the LISA method with those of endotracheal surfactant administration immediately followed by positive pressure ventilation with a designated lower peak pressure than it does in INSURE (LPPSA) in preterm infants with RDS and indicated for surfactant administration. Our hypothesis is that the effects of designated low positive pressure ventilation use following conventional surfactant administration (LPPSA) are not inferior to LISA in the treatment of preterm infants with RDS.

\section{Methods/design \\ Study aim}

This trial aims to compare the mortality and incidence of BPD at 36 weeks of PMA and the MV requirement during the first $72 \mathrm{~h}$ of life between LISA and LPPSA methods. 


\section{Setting}

The LISA Or Low Peak Pressure trial, a multicenter randomized prospective trial, will be conducted in 14 tertiary neonatal intensive care units (NICUs) in China from January 2019 to December 2020. The trial has been approved by the ethics committee of the Women's Hospital School of Medicine, Zhejiang University. This protocol has been registered in the Chinese Clinical Trial Registry (ChiCTR1900020970). The diagram of the study protocol is presented in Fig. 1.

\section{Grouping}

Infants will be divided into two groups, namely, the LISA group or LPPSA group, according to the method of surfactant administration. Infants in the LISA group will receive surfactants by using a thin catheter (LISA catheter ${ }^{\bullet}$ designed by Shuanghe Pharmaceutical, Beijing). Infants will be on nasal continuous positive airway pressure (NCPAP) support during the course of surfactant administration. Infants in the LPPSA group will be given surfactant by traditional intubation and MV support with a T-piece or ventilator immediately following surfactant administration as done in INSURE but with a peak inspiratory pressure at $<15 \mathrm{cmH}_{2} \mathrm{O} \quad(12-15$ $\mathrm{cmH}_{2} \mathrm{O}$ ), positive end-expiratory pressure (PEEP) at 6-8 $\mathrm{cmH}_{2} \mathrm{O}$, and respiratory rate at $40-60$ breaths per minute.

\section{Inclusion criteria}

Infants will be included if they meet all of the following criteria:

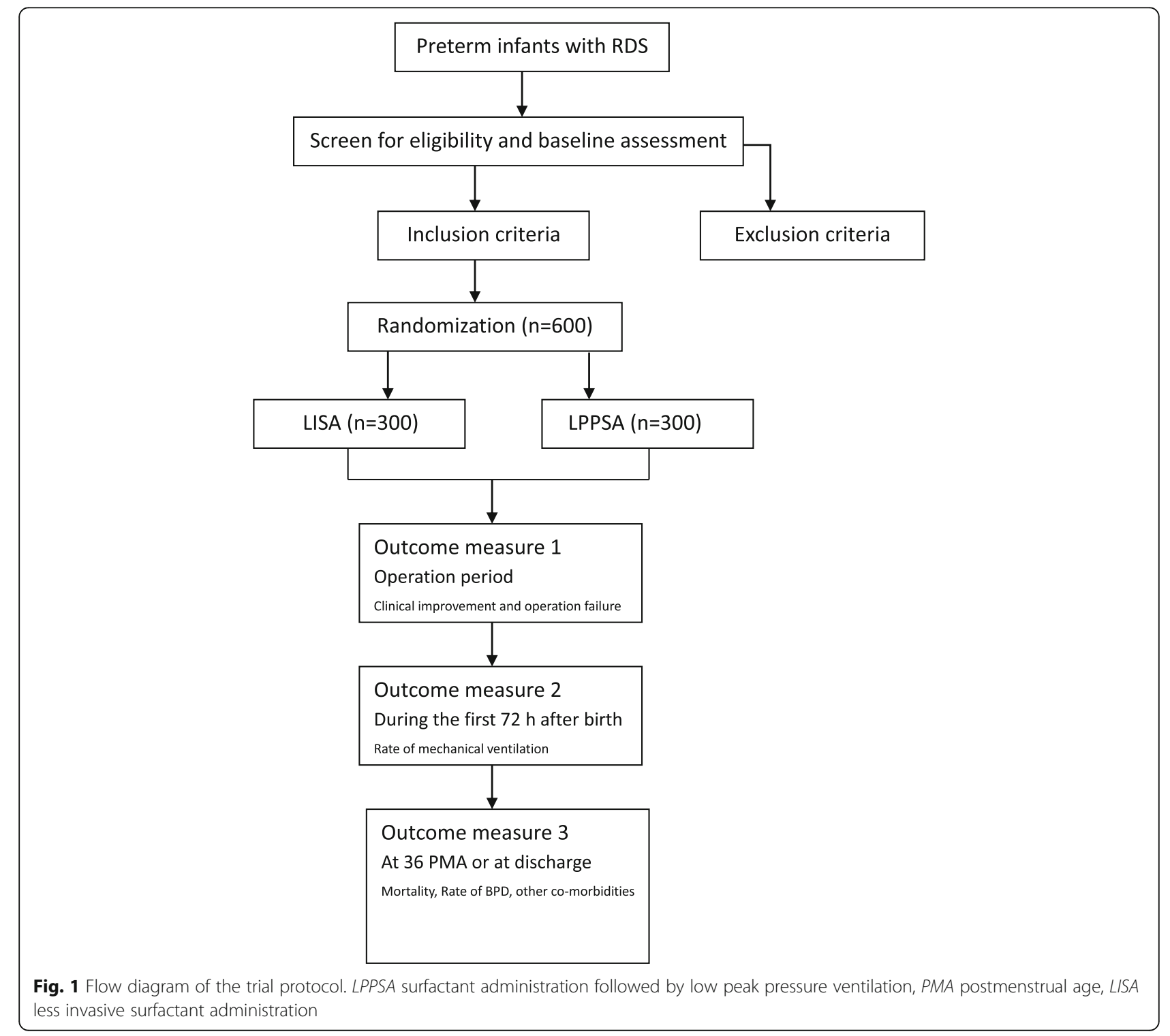


1. Born between $25^{0 / 7}$ and $31^{6 / 7}$ weeks of gestation with birth weight between $600 \mathrm{~g}$ and $1500 \mathrm{~g}$.

2. Exhibit vigorous spontaneous breathing and can be stabilized by noninvasive respiratory support

3. With PEEP at $6-8 \mathrm{cmH}_{2} \mathrm{O}$ and in need of fraction of inspired oxygen $\left(\mathrm{FiO}_{2}\right) \geqq 0.3$

4. Less than $6 \mathrm{~h}$ of age

5. Informed parental consent has been obtained

\section{Exclusion criteria}

Infants will be excluded if they meet any of the following:

1. Requiring MV or intubation in the delivery room

2. Presence of major congenital malformation or chromosomal abnormality or inherited disorders of metabolism

3. Have neuromuscular diseases affecting respiratory function

4. Presence of congenital pneumonia or pulmonary hypoplasia

\section{Randomization}

A randomization sequence with 600 numbers (odd to even numbers in a 1:1 ratio) will be generated by a computerized random number generator and placed in sealed opaque envelopes. Infants with odd and even numbers will be assigned to the LISA and LPPSA groups, respectively. Twins or multiple birth infants will be randomized separately. Every eligible infant will be allocated to each group according to a randomized sequence within $30 \mathrm{~min}$ by an independent person (a nurse in Women's Hospital School of Medicine, Zhejiang University).

\section{Blinding}

Operators and care providers will not be blinded, and the outcome assessors and data analysts will be blinded to the intervention. Decisions regarding ongoing treatments will be made according to every unit's guidelines and clinical practice. The authors had two meetings before the study to obtain consensus for neonatal care.

\section{Sample size calculation}

The proposed sample size is calculated according to the incidence of BPD. According to previous reports and the average data from every unit involved in the trials $[6,7]$, approximately $60 \%$ of infants born at $25^{+1}$ to $27^{+6}$ gestational weeks and approximately $15 \%$ among those born at $28^{+1}-31^{+6}$ gestational weeks have developed BPD during the past 5 years. The presumed incidence of $\mathrm{BPD}$ will be approximately $25 \%$ in the pooled population of our trial. Considering an alpha error rate of 0.05 and a power of 0.8 to detect an expected $20 \%$ reduction in the incidence of BPD, 258 infants will be enrolled in each group. To consider factors such as data incompleteness, failure to follow up, or early dropout, we plan to recruit 300 preterm neonates for each group.

\section{Intervention}

Positive pressure with a T-piece system (Neopuff Infant Resuscitation ${ }^{\oplus}$, Fisher and Paykel, Auckland, New Zealand) by a suitable mask will be applied to stabilize the infant after birth. Doctors in each participating center should be experienced using both methods. One must have experience with at least 10 cases implementing each technology.

\section{LISA method}

Oral sucrose will be encouraged during the whole procedure, and caffeine or any sedating drugs before operation will not be allowed in our trial. A specified thin catheter $(1.67 \mathrm{~mm}$ in diameter and $150 \mathrm{~mm}$ long, designed by Shuanghe Pharmaceutical, Beijing) is inserted beyond the vocal cords to the required depth (25-27 weeks at $1 \mathrm{~cm}, 28-31$ weeks at $1.5 \mathrm{~cm}$ ) under direct vision by laryngoscopy. Surfactant will be administered through the thin catheter within 2-3 min. During the procedure, the infant will be on NCPAP with a PEEP of 6-8 $\mathrm{cmH}_{2} \mathrm{O}$ and will receive tactile stimulation by the operator or nurse, who will rub the back or flick the sole, to maintain spontaneous breathing during the whole surfactant administration. The catheter will be immediately withdrawn after surfactant administration. If the infant develops apnea, bradycardia, or desaturation, surfactant administration will be stopped immediately. If the condition does not improve in $30 \mathrm{~s}$, a positive pressure ventilation will be given to recover the infants, as previously described $[1,6]$, and the case will be recorded as a failure of LISA.

\section{LPPSA method}

The same principle of premedication will be applied. Surfactant will be administered through an endotracheal tube under direct vision by laryngoscopy. Surfactant will be continuously administered through an endotracheal tube with mechanical ventilator or T-piece resuscitator. The initial setting will be as follows: PEEP of 6-8 $\mathrm{cmH}_{2} \mathrm{O}$, peak inspiratory pressure of $12-15 \mathrm{cmH}_{2} \mathrm{O}$, and respiratory rate of 40 breaths per minute. If the infant develops apnea, bradycardia, or desaturation, surfactant administration will be stopped immediately. If the condition does not improve in $30 \mathrm{~s}$, then conventional peak pressure ventilation $\left(18-20 \mathrm{cmH}_{2} \mathrm{O}\right.$ or more), will be given, and the case will be considered as a failure case of LPPSA. 


\section{Surfactant dosage}

Use of porcine surfactant (Curosurf ${ }^{\circledR}$, Chiesi, Italy) or bovine surfactant (Calsurf ${ }^{\ominus}$, Shuanghe, Pharmaceutical, Beijing) will be allowed in this trial. Based on literature concerned on the efficacy of different doses of surfactant and also from our experience, $200 \mathrm{mg} / \mathrm{kg}$ of porcine surfactant or $100 \mathrm{mg} / \mathrm{kg}$ of bovine surfactant will be used in the study. For infant with RDS as the primary cause, a second or third dose of $100 \mathrm{mg} / \mathrm{kg}$ (porcine) or $50 \mathrm{mg} / \mathrm{kg}$ (bovine) may be used if the infant's condition is deteriorated and RDS is still the primary consideration.

\section{CPAP and nasal intermittent positive-pressure ventilation (NIPPV)}

Both CPAP and NIPPV may reduce the morbidity of $\mathrm{BPD}$ in preterm infants when they are used as primary respiratory support. However, no consensus exists on the optimal setting value. In the trial, we use NCPAP as the primary noninvasive respiratory support during and after surfactant administration. The initial setting of PEEP will be at least $6 \mathrm{cmH}_{2} \mathrm{O}\left(6-8 \mathrm{cmH}_{2} \mathrm{O}\right)$. Both constant and variant flow NCPAP equipment are permitted to use in the trial based on the individual requirement. The proximal end of the gastric tube will be kept open to avoid abdominal distension, and soothing care such as use of pacifier or chin fixer can be used to achieve the expected pressure.

\section{MV indication}

The infant will receive MV therapy if any of the following condition occurs: (1) $\mathrm{FiO}_{2} \geqq 0.45$, PEEP $>8 \mathrm{cmH}_{2} \mathrm{O}$, $\mathrm{SPO}_{2}<90 \%$, and the condition lasts $>15 \mathrm{~min}$; (2) frequent or severe apnea even after the caffeine therapy. In the trial, $\geq$ four episodes of apnea requiring vigorous stimulation in $6 \mathrm{~h}$ is defined as frequent apnea, and severe apnea defined as $\geq 2$ episodes requiring positive pressure ventilation in $6 \mathrm{~h}$; (3) persistent (confirmed by two arterial blood gas samples at least $30 \mathrm{~min}$ apart) respiratory acidosis with $\mathrm{pH}<7.20$ and $\mathrm{PCO}_{2}>65 \mathrm{mmHg}$; (4) critical circumstances requiring intubation such as cardiac arrest caused by severe metabolic acidosis [8]. These criteria only apply to infants aged $\leq 1$ week, and will be used only for the first time after birth to judge whether MV management should be conducted instead of noninvasive ventilation.

\section{Indication for the weaning from MV}

The infant will be weaned from MV if all the following criteria are achieved. All preterm infants in this trial will receive caffeine therapy at least $24 \mathrm{~h}$ before weaning. Caffeine therapy with a loading dose of $20 \mathrm{mg} / \mathrm{kg}$ and then a maintenance dose of $5-10 \mathrm{mg} / \mathrm{kg}$ is encouraged in the trial. These criteria are as follows:
(1) The arterial blood gas should be maintained in a targeted range $\left(\mathrm{pH} \geqq 7.20, \mathrm{PaO}_{2} \geqq 50 \mathrm{mmHg}\right.$, $\mathrm{PaCO}_{2} \leqq 60 \mathrm{mmHg}$ ) with a low positive pressure (mean arterial pressure (MAP) $\leqq 7 \mathrm{cmH}_{2} \mathrm{O}, \mathrm{FiO}_{2}$ $\leqq 0.3)$.

(2) Stable spontaneous breathing should be present.

\section{Indication for noninvasive support weaning}

The infant will be weaned from NCPAP or NIPPV, if it has been $24 \mathrm{~h}$ since all of the following criteria will be achieved:

(1) $\mathrm{MAP} / \mathrm{PEEP} \leqq 3-5 \mathrm{cmH}_{2} \mathrm{O}$

(2) $\mathrm{FiO}_{2} \leqq 0.25$

(3) No apnea and bradycardia that requires stimulation

\section{Termination of the study}

(1) Death in hospitalization

(2) Early dropout on account of parents' decision

\section{Outcomes}

\section{Primary outcomes}

The primary outcomes will be as follows:

(1) Mortality, co-morbidity, and severity of BPD at 36 weeks of corrected gestational age.

(2) MV requirement in the first $72 \mathrm{~h}$ of life.

\section{Secondary outcomes}

The secondary outcomes will be as follows:

(1) Need for and duration of MV (days) during of hospitalization

(2) Duration of noninvasive respiratory support (days)

(3) Duration of oxygen need.

(4) Heart rate and oxygen saturation before and after surfactant administration in every $30 \mathrm{~s}$ for $10 \mathrm{~min}$.

(5) Incidence of pneumothorax and massive pulmonary hemorrhage within $48 \mathrm{~h}$ of surfactant administration

(6) Severe neonatal diseases including and hemodynamically significant patent ductus arteriosus (hsPDA) that needs medical or surgical intervention

(7) Duration of hospitalization

(8) Failure rate of operation (including LISA or LPPSA technique)

(9) Time required to perform the procedure of surfactant administration.

\section{Other data}

Other infant information that will be collected is as follows: gestational age, birth weight, sex, delivery mode, 
Apgar score at 1 and $5 \mathrm{~min}$, antenatal steroid use, prolonged premature rupture of membrane $(>18 \mathrm{~h})$, intraventricular hemorrhage (IVH, grade 3-4), stage II-III necrotizing enterocolitis (NEC), retinopathy of prematurity (ROP, $\geqq$ III stage), and postnatal steroid use.

\section{Definitions}

$B P D$

BPD is defined as postnatal treatment with oxygen > $21 \%$ for at least 28 days, plus oxygen requirement at 36 weeks PMA [9]. According to our inclusive criteria, the time point of the assessment is 36 weeks PMA or discharge to home, whichever comes first, and the grading criteria are as follows: (1) mild, breathing room air; (2) moderate, need for $<30 \%$ oxygen; and (3) severe, need for $\geq 30 \%$ oxygen and/or positive pressure support [9]. If the infant dies in the early days of life because of lethal BPD, a special case will be recorded separately according to revised definition of BPD [10].

\section{NEC (proven and advanced)}

Proven NEC (stage II) encompasses the signs of stage I plus the absence of bowel sounds with or without abdominal tenderness. Abdominal tenderness is present, and some infants have cellulitis of the abdominal wall or a mass in the right lower quadrant. Infants with stage IIA are mildly ill, whereas those with stage IIB NEC are moderately ill and have mild metabolic acidosis and thrombocytopenia. Findings on abdominal imaging include intestinal dilation, ileus, ascites, and pneumatosis intestinalis, which is the defining feature of stage II.

Advanced NEC (stage III) is the most severe form. In stage IIIA, the bowel is intact, whereas stage IIIB is characterized by bowel perforation visualized as a pneumoperitoneum on the abdominal radiograph. Infants with advanced NEC are critically ill. In addition to the signs shown in the less severe stages, they typically have hypotension, bradycardia, severe apnea, and signs of peritonitis (abdominal distention and marked tenderness). Laboratory signs include a combined respiratory and metabolic acidosis, neutropenia, and disseminated intravascular coagulation [11].

\section{IVH (grades III and IV)}

IVH with grade III is defined as IVH involving more than $50 \%$ of the ventricular area; lateral ventricles are usually distended. IVH with grade IV is characterized by hemorrhagic infarction in the periventricular white matter ipsilateral to IVH [12].

\section{Severe ROP}

Severe ROP, defined as ROP needing surgical intervention, including intravitreal injection, laser therapy, and cryotherapy, will be recorded in our study [13].

\section{Massive pulmonary hemorrhage}

This condition is characterized by the presence of hemorrhagic fluid from the endotracheal tube, accompanied by a sudden respiratory distress and deterioration of clinical condition (increased parameters or MV therapy is required with $1 \mathrm{~h}$ of the occurrence of blood fluid). X-ray imaging suggests involvement of more than two lung lobes [14].

\section{Pulmonary air leak in the newborn}

This condition is characterized by air leak from the lung identified by X-ray imaging, including pneumothorax, pneumomediastinum, pulmonary interstitial emphysema, and pneumopericardium.

\section{Adverse events}

Serious adverse events (SAEs) include death, prolonged hospitalization, and persistent disability, which are expected to be closely related to surfactant administration in the opinion of local medical investigators. SAEs will be reported within $24 \mathrm{~h}$ to the local Ethics Committee and Data and Safety Monitoring Committee (DSMC), and will be reported within 3 working days to coordinating centers. Any recommendations will be disseminated to local investigators. Adverse events are characterized by operation failure as described above or any clinical deterioration, including BPD, proven or advanced NEC, IVH (grades III and IV), severe ROP, massive pulmonary hemorrhage and pulmonary air leak, which are related to surfactant administration. AEs will be reported to the coordinating centers every month and will be closely monitored by the DSMC.

\section{Data collection}

All data will be collected from patient records. Data will be entered by the doctor who participated in the study of the individual NICU on a web-based electronic case record form and a written case report form. Access to the form will be protected by password, and infants may be identified by number only. A full-time coordinator will be responsible for monitoring the progress of the study and collecting feedback information from each unit. Data will be collected at the following schedule (Table 1).

\section{Data monitoring committee}

An independent Data Monitoring Committee (DMC) belonging to the Central Ethics Committee of Women's Hospital School of Medicine, Zhejiang University, has been established for the trial. This committee will perform interim data analysis, investigate compliance with the trial, and monitor adverse events. 
Table 1 Timeline of the study and clinical data collection

\begin{tabular}{|c|c|c|c|c|c|}
\hline \multirow[t]{3}{*}{ TIMEPOINT } & \multicolumn{5}{|c|}{ Study Period } \\
\hline & \multirow{2}{*}{$\begin{array}{l}\text { Enrollment } \\
\text { Birth to } 0\end{array}$} & \multirow{2}{*}{$\begin{array}{l}\text { Allocation } \\
0\end{array}$} & \multicolumn{3}{|l|}{ Post-allocation } \\
\hline & & & $\begin{array}{l}\text { Surfactant } \\
\text { administration } \\
\text { (-10min-+10min) }\end{array}$ & $\begin{array}{l}72 \text { hours } \\
\text { after } \\
\text { birth }\end{array}$ & $\begin{array}{l}\text { At } 36 \text { weeks } \\
\text { PMA or at } \\
\text { discharge }\end{array}$ \\
\hline \multicolumn{6}{|l|}{ Enrolment: } \\
\hline Eligibility screen & $x$ & & & & \\
\hline Informed consent & $x$ & & & & \\
\hline Allocation & & $x$ & & & \\
\hline \multicolumn{6}{|l|}{ Intervention } \\
\hline LISA & & & $x$ & $x$ & $x$ \\
\hline LPPSA & & & $x$ & $x$ & $x$ \\
\hline \multicolumn{6}{|l|}{ Assessments } \\
\hline $\begin{array}{l}\text { Primary outcomes } \\
\text { 1.Need for ventilation during the first } 72 \text { hours }\end{array}$ & & & & $x$ & \\
\hline $\begin{array}{l}\text { 2. Mortality } \\
\text { 3. BPD }\end{array}$ & & & & & $x$ \\
\hline $\begin{array}{l}\text { Secondary outcomes } \\
\text { Need for and the duration of MV (days), duration (days) of non-invasive } \\
\text { respiratory support, severe co-morbidities, pneumothorax, massive pul- } \\
\text { monary hemorrhage within } 48 \text { h of surfactant administration, } \\
\text { hemodynamically significant patent ductus arteriosus that needs medical } \\
\text { or surgical intervention, } \\
\text { Duration of hospitalization, } \\
\text { Failure rate of LISA or LPPSA }\end{array}$ & & & $x$ & $x$ & $x$ \\
\hline
\end{tabular}

LPPSA surfactant administration followed by low peak pressure, LISA less invasive surfactant administration

\section{Statistical methods}

All data will be analyzed using SPSS version 20.0. To compare primary and secondary outcomes between the two groups, Student's t-test (continuous variables) and the chi-squared test (categorical variables) will be used. The two-level hierarchical linear regression model or the logistic regression model, whichever is appropriate, will be applied for the comparison of the outcomes between the two groups, accounting for the infants' characteristics, such as gestational age, birthweight, sex, appropriateness for gestational age, delivery mode, and pregnancy complications, and center variables, such as number of beds in the NICU and the number of infants with gestational age $<32$ weeks. $P<0.05$ will be regarded as statistically significant.

\section{Discussion}

In recent years, the LISA method has been accepted by increasing number of doctors. However, the potential benefit of LISA is still not clear. Moreover, an increased incidence of spontaneous intestinal perforation in extremely premature infants has been reported [15]. A meta-analysis showed that LISA showed effects on reducing the rate of MV during the first $72 \mathrm{~h}$ and the combination of BPD mortality and morbidity based on previous trials, which compared the effects of LISA with conventional surfactant technique (including intubation and ventilation by bagging or ventilator). We found that previous studies focused more on the procedure of performing LISA but less on the clarity of the potential mechanism [16, 17]. In fact, pulmonary compliance will change during the period of surfactant administration, and the peak pressure of ventilation barely changed spontaneously, even with use of a more advanced ventilator. A preliminary idea is that the whole procedure of surfactant administration without ventilation may contribute most to the advantages of LISA [18]. Thus, if correct, we would have further comprehension of LISA, and further knowledge would encourage more efforts on applying optimal peak pressure to surfactant delivery instead of trying to require all doctors to learn the method of LISA in China.

The delivery of surfactant as soon as possible has been known to help the distribution of the surfactant [19] In a previous study, the LISA procedure may took $0.5-5$ min (average 2-3 min), which is longer than that of conventional method. Desaturation and apnea are the main complications of LISA [20, 21]. This situation is seldom seen in conventional surfactant administration. The contradiction of "time and effects" between LISA and conventional method may suggest some potential benefits of LISA. In this trial, the time required to perform the procedure would also be evaluated, because of the variable data of previous studies.We hope it will give us 
more information of the reason and a relatively standard operation of LISA can be set.

To our knowledge, this trial will be the first multicenter randomized controlled trial to evaluate the potential benefits of LISA and will give a more objective evaluation of LISA.

\section{Trial status}

The protocol version is 1.0, and the issue date is 18 December 2018. Recruitment of participants started in January 2019 and is ongoing. Recruitment of participants is expected to end no later than December 2020.

\section{Supplementary information}

Supplementary information accompanies this paper at https://doi.org/10. 1186/s13063-020-04390-3.

\section{Additional file 1.}

\section{Abbreviations}

BPD: Bronchopulmonary dysplasia; $\mathrm{FiO}_{2}$ : Fraction of inspired oxygen; ICH: Intraventricular hemorrhage; MAP: Mean arterial pressure; MV: Mechanical ventilation; NCPAP: Nasal continuous positive airway pressure; NEC: Necrotizing enterocolitis; NICU: Neonatal intensive care unit; PEEP: Positive end-expiratory pressure; RDS: Respiratory distress syndrome; ROP: Retinopathy of prematurity; LISA: Less invasive surfactant administration; LPPSA: Low peak pressure surfactant administration

\section{Acknowledgements}

The authors would like to thank all families whose infants will participate in this trial and all staff providing patient care in all participating units. We thank our colleagues who have contributed to the study for recruitment, administrative help, and other advice. We also thank Editage (www.editage. com) for English language editing.

\section{Authors' contributions}

JZ and YB conceptualized the study and drafted the initial study protocol, LD participated in the study design and recruitment of other centers to participate in this trial, JZ and LD participated in revising and amending the protocol and approved the final version of the manuscript. All authors of the LOLPP study group read and approved the final version and agreed to participate in this study. All authors read and approved the final manuscript.

\section{Funding}

This study is supported by a grant from the Major scientific and technological projects of medical and health in Zhejiang Province (WKJ-ZJ2032) for the analysis and interpretation of data by a third party and also for the submission and publication of the manuscript.

\section{Availability of data and materials}

Not applicable.

\section{Ethics approval and consent to participate}

The trial protocol, informed consent forms, and other requested documents have been reviewed and approved by the Central Ethics Committee of Women's Hospital School of Medicine, Zhejiang University (case number: 20180155). We will not begin recruiting at other centers in the trial until local ethical approvals have been obtained. The progress and the safety of the study will be accessed every 6 months by the Central Ethics Committee of Women's Hospital School of Medicine, Zhejiang University. The written consent will be obtained from all participants. The trial was registered at Chinese Clinical Trial Registry on 23 January 2019 (ID: ChiCTR1900020970). Important protocol modifications will be communicated to these agencies. We respect the parents' decisions to join or leave the study at any time.

\section{Consent for publication}

The parents will sign the consent for publication.

\section{Competing interests}

The authors declare that they have no competing interests.

\section{Author details}

'Women's Hospital, Zhejiang University, School of Medicine, Hangzhou 310006, China. ${ }^{2} T h e$ Children's Hospital, Zhejiang University, School of Medicine, Hangzhou 310052, China. ${ }^{3}$ Jiaxing Maternity and Child Health Care Hospital, Jiaxing 314051, China. ${ }^{4}$ Ningbo Maternal and Children Hospital, Ningbo 315012, China. ${ }^{5}$ Shao Xing Maternity and Child Health Care Hospital, Shaoxing 312000, China. ${ }^{6}$ Central Hospital of Jinhua, Jinghua 321000, China. ${ }^{7}$ Boai Hospital of Zhongshan, Zhongshan 528400, China. ${ }^{8}$ Gansu Provincial Maternity and Child-Care Hospital, Lanzhou 730050, China. ${ }^{9}$ Shengjing Hospital of China Medical University, Shenyang 110004, China. ${ }^{10}$ Affiliated Shenzhen Maternity\& Child Healthcare Hospital, Southern Medical University, Shenzhen 518028, China. ${ }^{11}$ Affiliated Hospital of Inner Mongolia Medical University, Hohhot 010050, China. ${ }^{12}$ Yan'an University Affiliated Hospital, Yan'an 716000, China. ${ }^{13}$ Chongqing Health Center for Women and Children, Chongqing 400021, China. ${ }^{14}$ Linyi People's Hospital, Linyi 276003, China.

Received: 26 December 2019 Accepted: 8 May 2020

Published online: 11 June 2020

References

1. Sweet DG, Carnielli V, Greisen G, Hallman M, Ozek E, Te Pas A, et al. European consensus guidelines on the management of respiratory distress syndrome - 2019 update. Neonatology. 2019;115:432-50.

2. Banerjee S, Fernandez R, Fox GF, Goss KCW, Mactier H, Reynolds P, et al. Surfactant replacement therapy for respiratory distress syndrome in preterm infants: United Kingdom national consensus. Pediatr Res. 2019;86:12-4.

3. Lista G, Bresesti I, Fabbri L. Is less invasive surfactant administration necessary or "only" helpful or just a fashion? Am J Perinatol. 2018;35:530-3.

4. Aldana-Aguirre JC, Pinto M, Featherstone RM, Kumar M. Less invasive surfactant administration versus intubation for surfactant delivery in preterm infants with respiratory distress syndrome: a systematic review and metaanalysis. Arch Dis Child Fetal Neonatal Ed. 2017;102:F17-23.

5. Herting E, Härtel C, Göpel W. Less invasive surfactant administration (LISA): chances and limitations. Arch Dis Child Fetal Neonatal Ed. 2019;104:F655-F9.

6. Bao Y, Zhang G, Wu M, Ma L, Zhu J. A pilot study of less invasive surfactant administration in very preterm infants in a Chinese tertiary center. BMC Pediatr. 2015;15:21.

7. Lin HJ, Du LZ, Ma XL, Shi LP, Pan JH, Tong XM, et al. Mortality and morbidity of extremely low birth weight infants in the mainland of China: a multi-center study. Chin Med J. 2015;128(20):2743-50.

8. Lee BK, Shin SH, Jung YH, Kim EK, Kim HS. Comparison of NIV-NAVA and NCPAP in facilitating extubation for very preterm infants. BMC Pediatr. 2019; 19:298.

9. Jobe $A H$, Bancalari E. Bronchopulmonary dysplasia. Am J Respir Crit Care Med. 2001;163:1723-9.

10. Higgins RD, Jobe AH, Koso-Thomas M, Bancalari E, Viscardi RM, Hartert TV, et al. Bronchopulmonary dysplasia: executive summary of a workshop. J Pediatr. 2018;197:300-8.

11. Bell MJ. Neonatal necrotizing enterocolitis. N Engl J Med. 1978;298:281-2.

12. Papile LA, Burstein J, Burstein R, Koffler H. Incidence and evolution of subependymal and intraventricular hemorrhage: a study of infants with birth weights less than 1,500 gm. J Pediatr. 1978;92:529-34.

13. Dhingra D, Katoch D, Dutta S, Samanta R, Aggarwal K, Dogra MR. Change in the incidence and severity of retinopathy of prematurity (ROP) in a neonatal intensive care unit in Northern India after 20 years: comparison of two similar prospective cohort studies. Ophthalmic Epidemiol. 2019;26:169-74.

14. Kanavaki I, Vutskits L, Rimensberger PC. Use of therapeutic surfactant lavage in a preterm infant with massive pulmonary hemorrhage. Clin Pract. 2012;2: e74.

15. Härtel C, Paul P, Hanke K, Humberg A, Kribs A, Mehler K, et al. Less invasive surfactant administration and complications of preterm birth. Sci Rep. 2018; 8:8333.

16. Göpel W, Kribs A, Härtel C, Avenarius S, Teig N, Groneck P, et al. Less invasive surfactant administration is associated with improved pulmonary 
outcomes in spontaneously breathing preterm infants. Acta Paediatr. 2015; 104:241-6.

17. Herting E. Less invasive surfactant administration (LISA) - ways to deliver surfactant in spontaneously breathing infants. Early Hum Dev. 2013;89:875-80

18. Niemarkt HJ, Kuypers E, Jellema R, Ophelders D, Hütten M, Nikiforou M, et al. Effects of less-invasive surfactant administration on oxygenation, pulmonary surfactant distribution, and lung compliance in spontaneously breathing preterm lambs. Pediatr Res. 2014;76:166-70.

19. Kerr CL, Ito Y, Manwell SE, Veldhuizen RA, Yao LJ, McCaig LA, et al. Effects of surfactant distribution and ventilation strategies on efficacy of exogenous surfactant. J Appl Physiol (1985). 1998:85:676-84.

20. Klotz D, Porcaro U, Fleck T, Fuchs $H$. European perspective on less invasive surfactant administration-a survey. Eur J Pediatr. 2017;176:147-54.

21. Vento M, Bohlin K, Herting E, Roehr CC, Dargaville PA. Surfactant Administration via thin catheter: a practical guide. Neonatology. 2019;116(3): $211-26$

\section{Publisher's Note}

Springer Nature remains neutral with regard to jurisdictional claims in published maps and institutional affiliations.

Ready to submit your research? Choose BMC and benefit from:

- fast, convenient online submission

- thorough peer review by experienced researchers in your field

- rapid publication on acceptance

- support for research data, including large and complex data types

- gold Open Access which fosters wider collaboration and increased citations

- maximum visibility for your research: over $100 \mathrm{M}$ website views per year

At $\mathrm{BMC}$, research is always in progress.

Learn more biomedcentral.com/submissions 